\title{
Downward mapping of quasi-static ionospheric electric fields at low latitudes during fair weather
}

\author{
S. L. G. Dutra, ${ }^{*}$ A. L. C. Gonzalez, ${ }^{*}$ W. D. Gonzalez* and A. E. C. Pereira $\dagger$ \\ * Instituto Nacional de Pesquisas Espaciais-INPE, C.P. 515, 12201 - São José dos Campos. \\ SP, Brazil; \\ † Universidade Federal de Uberlândia-UFU, Departamento de Engenharia Elétrica, 38400 . \\ Uberlândia, MG, Brazil
}

(Received in final form 27 March 1991 )

\begin{abstract}
The problem of downward mapping of equatorial ionospheric electric fields is studied in two dimensions employing the finite elements and finite differences numerical techniques. The solutions obtained for low latitudes are compared with known results for high latitudes. It is found that equatorial ionospheric electric fields of scale lengths of the order of $100 \mathrm{~km}$ or more reach balloon heights $(30-40 \mathrm{~km})$ without undergoing noticeable attenuation. However, in the case of equatorial ionospheric electric fields of scale lengths of a few tens of kilometers it is found that these fields reach balloon heights with severe attenuation. The corresponding attenuation factors are significantly larger than those known for high latitudes. It is also shown that the presence of mountains with a fairly large height as well as of a large-scale conductivity irregularity in the middle atmosphere, such as that expected in the South Atlantic Magnetic Anomaly (SAMA) region during energetic electron precipitation events, can considerably distort the mapped ionospheric electric fields at the middle atmosphere.
\end{abstract}

\section{INTRODUCTION}

Several authors have already studied the problem of downward mapping of ionospheric electric fields at high latitudes (e.g. Mozer, 1971; Dejnakarintra, 1974; ChIU, 1974; Park, 1976, 1979). On the other hand, besides the initial study by VoLLAND (1972) of downward mapping of ionospheric electric fields at equatorial latitudes, not much work has been done. This is in spite of the considerable knowledge already accumulated on ionospheric electric fields at equatorial and low latitudes (e.g. WOODMAN, 1970; GonZales $e t$ al., 1979; Richmond et al., 1980).

At the equatorial latitudes the geomagnetic field is almost horizontal in contrast to the practically vertical one at high latitudes. We expect that this geometrical factor can introduce a basic difference in the mapping problem through the effective role of the conductivities along and transverse to the magnetic field, at least above about $80 \mathrm{~km}$ altitude where the conductivity tensor is anisotropic. Furthermore, even at lower altitudes we expect additional differences in the mapping study between equatorial and high latitudes, sinec it is known that the corresponding isotropic conductivity profiles are also different, mainly due to the latitudinal effect of cosmic ray penetration (e.g. SANDSTRÖM, 1965).

Thus, in the present work an example of the equatorial mapping study for quasi-static electric fields during a fair weather period is presented in two dimen- sions. An additional study of influences due to orographic electric fields and to an atmospheric conductivity irregularity are also included (Durra, 1982). The calculations refer only to horizontal ionospheric electric fields for the noontime. An atmospheric mean potential difference of $300 \mathrm{kV}$ between ground and ionosphere representing an unperturbed and average fair weather electric field is assumed.

Although the magnitudes of the equatorial ionospheric elcctric ficlds (of the order of a few millivolts per meter) are much smaller than those at the high latitude ionosphere, the knowledge of their extension to the lower atmosphere becomes necessary, mainly when electric fields measured in the atmosphere are compared to the overhead ionospheric field. Efforts of this sort have already been made (Gonzalez et $\mathrm{al}$., 1982, 1987) through electric field measurements at balloon altitudes in the low latitude (almost equatorial) atmosphere.

\section{THEORY AND RESULTS}

The basic equations that describe quasi-static electric processes in the atmosphere are:

$$
\begin{gathered}
\nabla \cdot \mathbf{J}=0 \\
\mathbf{J}=\tilde{\sigma} \cdot \mathbf{E} \\
\mathbf{E}=-\nabla \phi
\end{gathered}
$$


together with appropriate boundary conditions. The vectors $\mathbf{J}$ and $\mathbf{E}$ are the electric current density and the electric field, respectively. The tensor $\tilde{\sigma}$ is the electric conductivity tensor and the scalar $\phi$ is the electric potential for the assumed irrotational electric field.

Consider a cartesian coordinate system $(x, y, z)$ with positive $x$-axis pointing towards the south, positive $y$ towards the east and positive $z$ upwards. For this system equations (1)-(3) give :

$$
\begin{aligned}
& \left(\sigma_{0} C^{2}+\sigma_{1} S^{2}\right) \frac{\hat{\sigma}^{2} \phi}{\partial x^{2}}+\sigma_{1} \frac{\partial^{2} \phi}{\partial y^{2}}+\left(\sigma_{0} S^{2}+\sigma_{1} C^{2}\right) \frac{\partial^{2} \phi}{\partial z^{2}} \\
& +2\left(\sigma_{0}-\sigma_{1}\right) S C \frac{\partial^{2} \phi}{\partial x \partial y}+\left[\frac{\partial \sigma_{0}}{\partial x} C^{2}+\frac{\partial \sigma_{t}}{\partial x} S^{2}\right. \\
& \left.-\frac{\partial \sigma_{2}}{\partial y} S+\left(\begin{array}{c}
\partial \sigma_{0} \\
\partial z
\end{array}-\frac{\partial \sigma_{1}}{\partial z}\right) S C\right] \frac{\partial \phi}{\partial x}+\left(\frac{\partial \sigma_{2}}{\partial x} S+\frac{\partial \sigma_{1}}{\partial y}\right. \\
& \left.-\frac{\partial \sigma_{2}}{\partial z} C\right) \frac{\partial \phi}{\partial y}+\left[\left(\frac{\partial \sigma_{0}}{\partial x}-\frac{\partial \sigma_{1}}{\partial x}\right) S C+\frac{\partial \sigma_{2}}{\partial y}\right. \\
& \left.+\frac{\partial \sigma_{0}}{\partial z} S^{2}+\frac{\partial \sigma_{1}}{\partial z} C^{2}\right] \frac{\partial \phi}{\partial z}=0
\end{aligned}
$$

with the conductivity tensor $\tilde{\sigma}$ given by:

$$
\tilde{\sigma}=\left(\begin{array}{ccc}
\sigma_{1} S^{2}+\sigma_{0} C^{2} & \sigma_{2} S & \left(\sigma_{0}-\sigma_{1}\right) S C \\
-\sigma_{2} S & \sigma_{1} & \sigma_{2} C \\
\left(\sigma_{0}-\sigma_{1}\right) S C & -\sigma_{2} C & \sigma_{1} C^{2}+\sigma_{0} S^{2}
\end{array}\right)
$$

where $\sigma_{0}, \sigma_{1}$ and $\sigma_{2}$ are the direct, Pedersen and Hall conductivities, respectively. Other parameters are defined by $S=\sin I, C=\cos I$, where $I$ is the dip angle of the geomagnetic field $\left(I=0^{\circ}\right.$ at the equator and $I=90$ at the poles). Figure 1 is an example of conductivity profiles for the equatorial atmosphere at noon (WeBb, 1974; Forbes and LindzEN, 1976). A direct conductivity profile due to COLE and Pierce (1965) is also shown in Fig. 1. Note that the $\sigma_{2}$ profile has a sharp change at about $70 \mathrm{~km}$.

From equation (4) and for regions where there are no horizontal gradients we obtain by direct integration:

$$
\phi(z)=\frac{\phi\left(z_{m}\right)}{R_{m}} \int_{0}^{z} \frac{\mathrm{d} z}{\sigma_{0} S^{2}+\sigma_{1} C^{2}}
$$

where $z_{m}$ is a reference height with $\phi\left(z_{m}\right)>0$ (see discussion below) and $R_{m}$ is the atmospheric columnar resistance

$$
R_{m}=\int_{0}^{-m} \frac{\mathrm{d} z}{\sigma_{0} S^{2}+\sigma_{1} C^{2}}
$$

Besides the implicit variation of the $\sigma_{0}(z)$ and $\sigma_{1}(z)$

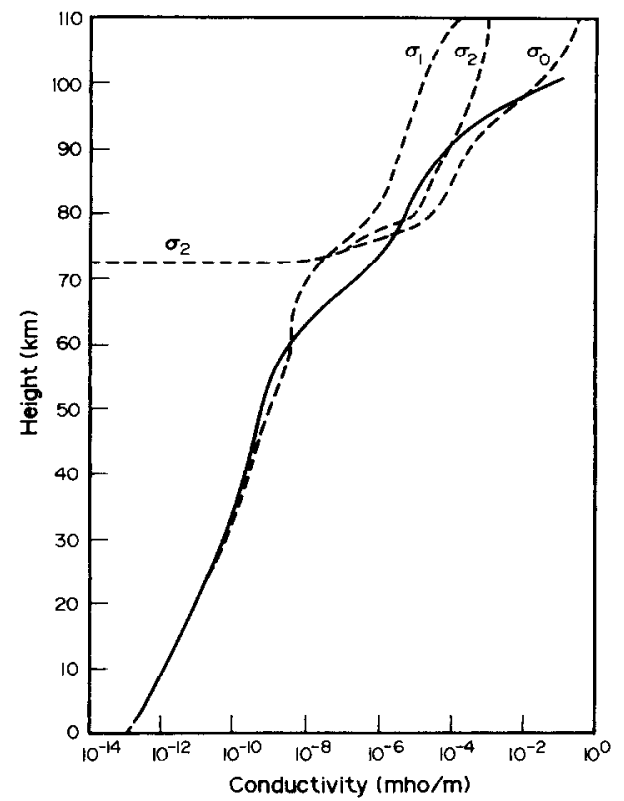

Fig. 1. Profiles for the electric conductivities at the equatorial noon atmosphere, as obtained from WEBB (1974) and FORBES and Lindzen (1976). The full line curve is due to CoLf and Pierce (1965).

profiles with latitude the new result given by equation (6) generalizes the atmospheric fair weather potential $\phi(z)$ calculation at any latitude through the explicit role of the conductivities and the dip angle (by the factors $S$ and $C$ ) variations. Note that $\sigma_{0} S^{2}+$ $\sigma_{1} C^{2}=\sigma$ for altitudes with isotropic conductivity $\sigma$ (since $S^{2}+C^{2}=1$ ), and we have only the intrinsic conductivity variation with latitude in the characterization of $\phi(z)$. With a uniform ionospheric potential $\phi\left(z_{m}\right)$, the vertical electric field (points downward) is given by

$$
E_{z}=-\frac{\phi\left(z_{m}\right)}{R_{m}} \frac{1}{\sigma_{0} S^{2}+\sigma_{1} C^{2}}
$$

Our mapping study at the equator (two dimensional) will restrict to the $(y, z)$ plane, because the large-scale ionospheric electric fields of interest lie in the east-west direction in this region. For this plane, equation (4) reduces to:

$$
\begin{aligned}
\sigma_{1} \frac{\partial^{2} \phi}{\partial y^{2}}+\sigma_{1} \frac{\partial^{2} \phi}{\partial z^{2}}+\left(\frac{\partial \sigma_{1}}{\partial y}-\frac{\partial \sigma_{2}}{\partial z}\right) \frac{\partial \phi}{\partial y} \\
+\left(\frac{\partial \sigma_{2}}{\partial y}+\frac{\partial \sigma_{1}}{\partial z}\right) \frac{\partial \phi}{\partial z}=0 .
\end{aligned}
$$

At high latitudes, the corresponding equation (PARK, 
1976) obtained from (4) is :

$$
\sigma_{1} \frac{\partial^{2} \phi}{\partial x^{2}}+\sigma_{0} \frac{\partial^{2} \phi}{\partial z^{2}}+\frac{\partial \sigma_{1}}{\partial x} \frac{\partial \phi}{\partial x}+\frac{\partial \sigma_{0}}{\partial z} \frac{\partial \phi}{\partial z}=0 .
$$

Note that, while equation (7) is governed by the Pedersen $\sigma_{1}$ and Hall $\sigma_{2}$ conductivities, equation (8) is governed by the direct $\sigma_{0}$ and Pedersen $\sigma_{1}$ conductivities.

For the study of the fair weather vertical electric field (DOLEZALEK, 1972) only, we assume that there are no horizontal gradients present. For this case equation (6) reduces to:

$$
\phi(z)=\phi\left(z_{m}\right) \int_{0}^{z} \begin{gathered}
\mathrm{d} z \\
\sigma_{1}
\end{gathered} / \int_{0}^{z_{m}} \frac{\mathrm{d} z}{\sigma_{1}}
$$

where $z_{m}$ is usually taken at the top of the atmosphere (ionosphere). Note that $\phi(0)-0$. The value of $\phi\left(z_{m}\right)$ can be assumed to be $300 \mathrm{kV}$, the usual value of the average fair weather potential at the ionospheric height. Thus, once $\sigma_{1}(z)$ is specified, equation (9) gives the potential profile for the equatorial atmosphere. For high latitudes studies it is interesting to note that the atmospheric potential profile is governed by the $\sigma_{0}(z)$ profile (PARK, 1976) in contrast to the equatorial case where we find the Pedersen conductivity in the same role, namely from equation (6) :

$$
\phi(z)=\phi\left(z_{m}\right) \int_{0}^{z} \frac{\mathrm{d} z}{\sigma_{0}} / \int_{0}^{z_{m}} \frac{\mathrm{d} z}{\sigma_{0}} .
$$

The magnitude of the vertical electric field $E_{z}(z)$ is inversely proportional to $\sigma_{1}(z)$ at the equatorial atmosphere. At high latitudes $E_{z}(z)$ is inversely proportional to $\sigma_{0}(z)$.

In order to solve equation (7) for modelling the ionospheric mapping, the boundary conditions of interest are : $\phi=0$ and $z=0 ; \phi_{m}(y)=\phi\left(y, z_{m}\right)$ is specified at $z=z_{m}$ (ionospheric level, which in this work will be assumed at $110 \mathrm{~km}$ altitude); and $\phi(y \rightarrow$ $\pm \infty, z)$ is given by equation (9), representing the fair weather electric field at distances far from the origin, where horizontal gradients are assumed to vanish.

The assumed uniform ionospheric electric field $E_{y 0}$, confined in a region of width $L$ between $y=-L / 2$ and $y=L / 2$ at $z=z_{m}$, and the atmospheric potential $\phi_{m}(y)$ are shown in Fig. 2. This assumption allows to model a constant horizontal electric field parameterized with a scale length $L$, representing the existing large scale electric field of ionospheric origin. Within the scope of this mapping study the adopted form of $\phi_{m}(y)$ is sufficient for our purposes and it is also computationally efficient. Since the fair weather vertical electric field is always present, a typical poten-

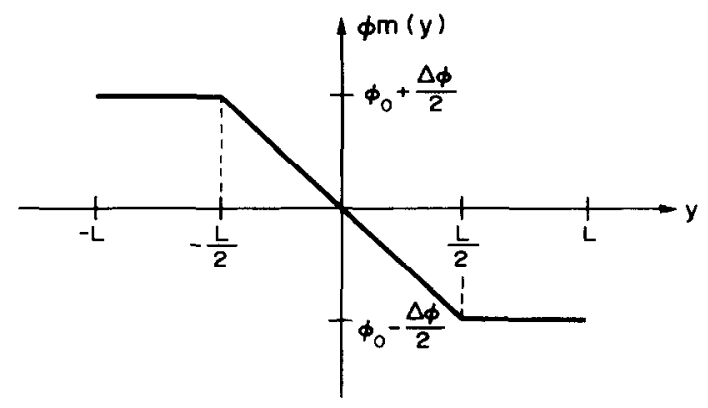

Fig. 2. Model of atmospheric potential representing an ionospheric electric field of scale length $L$ superimposed to the fair weather field (with ionospheric electric potential $\phi_{0}$ ).

tial difference $\phi_{0}=300 \mathrm{kV}$ exists between the ground and ionosphere. Equation (7) can be solved numerically with the finite difference method and a consistently ordered line scheme (DUTRA, 1982; DutrRA et al., 1985). A summary of this solution (BTM method) is indicated in the Appendix.

Figure 3 shows the curves for the attenuated values of the atmospheric electric field, obtained for $y=0$ and for selected values of the horizontal seale length $L$. Although the ionospheric electric field $E_{p 0}$ always decreases with decreasing altitude, its attenuation is a strong function of $L$. Thus, whereas the electric field is strongly attenuated already at the top of the atmosphere for $L=10 \mathrm{~km}$, the attenuation is negligible through most of the atmosphere for $L \geqslant 100 \mathrm{~km}$. The later result is similar to that found for high latitudes (e.g. Mozer, 1971; PArk, 1976). The dashed curves in Fig. 3 are two examples for high latitudes (PARK, 1976). Note that for $L$ values of the order of tens of kilometers, as for the illustrated case of $L=20 \mathrm{~km}$, ionospheric fields are more attenuated at the equator than at high latitudes.

We should note that the results shown in Fig. 3 are valid only for atmospheric altitudes above about 10 $\mathrm{km}$. At lower altitudes the (horizontal) atmospheric electric field is rapidly attenuated, regardless of the magnitude of $L$ (PARK, 1976; PARK and DEJNAKARINTRA, 1973), because of the cancelling effect of electric fields arising from induced charges on the Earth's surface. Figure 4 shows the horizontal variation of the attenuated electric field at balloon altitudes ( $z$ around $30 \mathrm{~km}$ ), normalized with respect to $E_{y 0}$. Again, the curves of this figure give similar results to those found for high latitudes (PARK, 1976) when $L \geqslant 100 \mathrm{~km}$, whereas notable differences exist for $L$ values of the order of tens of kilometers, with the attenuation being larger at the equator than at high latitudes for the corresponding values of $L$. The map- 


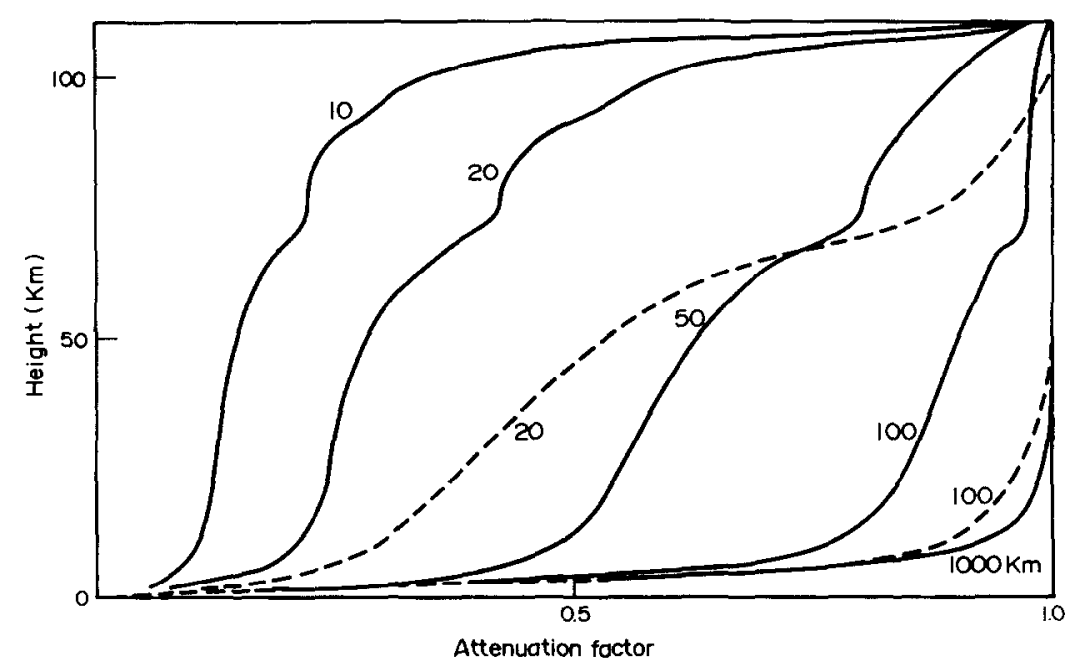

Fig. 3. Curves for the attenuated atmospheric electric field, at $y=0$, for several scale length $L$ values of the ionospheric electric field. The dashed curves refer to high latitudes (PARK, 1976).

ping reproduces the fair weather electric field, as expected, for points very far from the origin (characterized with a null magnitude of the horizontal component), with the ratio $E_{y} / E_{y 0}$ going to zero independent of the magnitude of $L$ when $y / L \gg 1$. Recall that for these regions, near the edges, equation (9) must be obeyed. Again the dashed curves represent two examples for comparison with the high latitude study.

During fair weather, atmospheric sources like large orographic features (ATKINSON et al., 1971; OGAWA et al., 1975) and large-scale conductivity perturbations in the middle atmosphere (GonzalEz et al., 1987) are

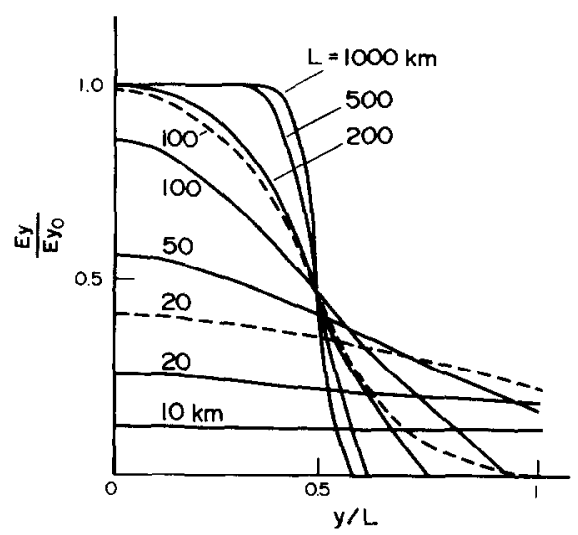

Fig. 4. Curves for the horizontal variation of the attenuated electric field at balloon altitudes, for several values of the scale length $L$, and normalized with respect to the ionospheric electric field $E_{y 0}$. The dashed curves refer to high latitudes (PARK, 1976). expected to affect the above described mapping study.

Considering simple boundary conditions for the electric potential, just representing the fair weather electric field, Fig. 5 shows the horizontal electric fields produced by a mountain with height $h$ and base $b$ as curves of equal electric field values varying with height. These curves were automatically plotted for cases: (a) $h=5 \mathrm{~km}, b=52 \mathrm{~km}$, and (b) $h=1 \mathrm{~km}$, $b=10 \mathrm{~km}$. We clearly observe that bigger mountains produce larger influences in the fair weather atmospheric electric field (purely vertical) than smaller ones. We see from these figures that for a measurement point situated, for example, $60 \mathrm{~km}$ away from the mountain and at a balloon altitude of $30 \mathrm{~km}$, the presence of the mountains in the measured values (perturbation in the horizontal electric field) has a magnitude around $2 \mathrm{mV} / \mathrm{m}$ in case (a) and around $0.05 \mathrm{mV} / \mathrm{m}$ in case (b). The direction of this perturbation is toward east for points situated west of the mountain and vice versa. Due to the need to select an appropriate discretization grid, that can be adjustable to the presence of mountains, the finite element method was used in this calculation (Appendix).

Another influence on the mapped ionospheric electric field is related to the irregularities in the atmospheric electrical conductivity originated by energetic charged particle precipitation, producing distortions of the fair weather atmospheric electric field. For high latitudes the effect of some of those irregularities has becn studicd (PARK, 1976) and a rclated large modification of the stratospheric electric field event has been observed during the strong solar flare of August 1972 (Holzworth and Mozer, 1979). At low latitudes 


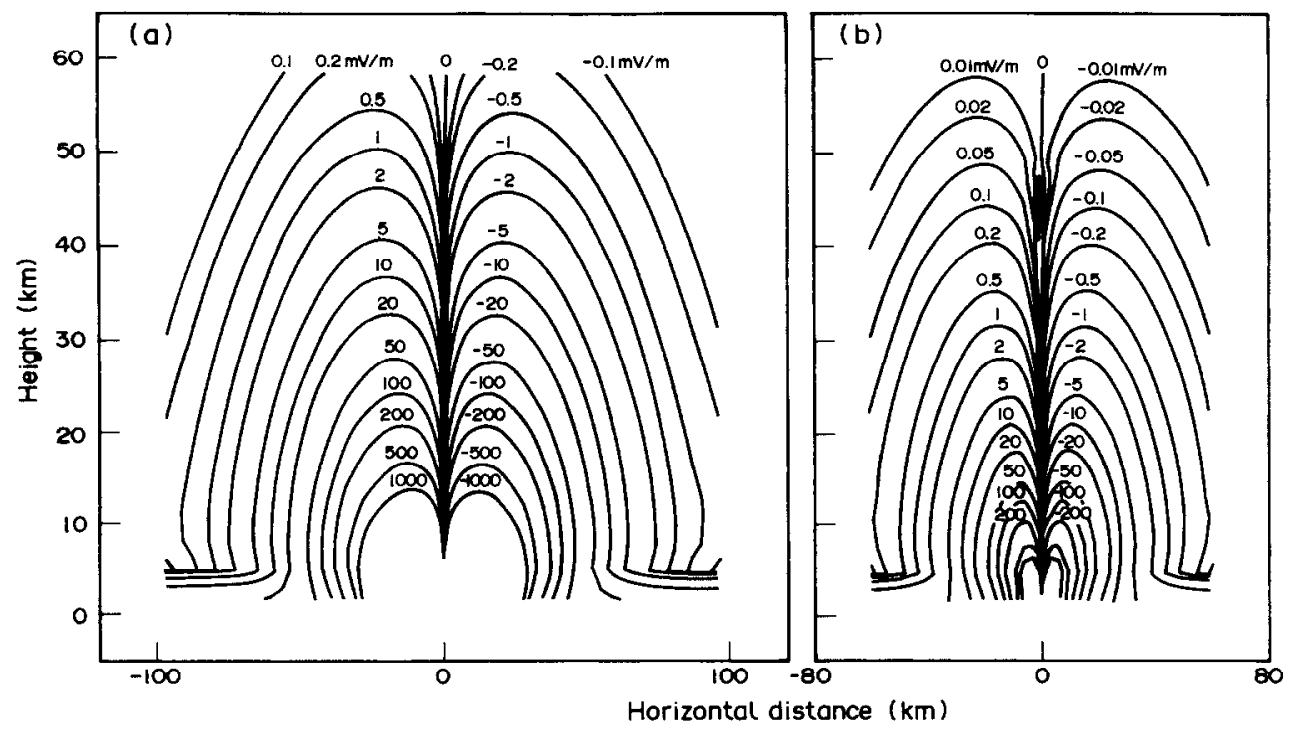

Fig. 5. Horizontal electric fields due to a mountain (a) with $5 \mathrm{~km}$ height and $5 ? \mathrm{~km}$ base and (h) with $1 \mathrm{~km}$ height and $10 \mathrm{~km}$ base.

and, particularly, in the South Atlantic Magnetic Anomaly (SAMA) region, energetic electron precipitation in the atmosphere is also believed to create a conductivity enhancement at the middle atmosphere and distort the fair weather electric field at stratospheric levels (GonZALEZ et al., 1982, 1987).

To model this effect the irregularity is assumed to be centered at $y=0$ and at $z=z_{i}$ (middle atmosphere), and characterized by an amplification factor $f$ (above normal values) given by [following PARK (1976) and Dutra (1982)] :

$$
f(y, z)=\left\{\begin{array}{cl}
1+(\Lambda-1) \exp \left[-\left(\frac{z-z_{i}}{z_{0}}\right)^{2}\right], & \text { if }|y| \leqslant Y_{0} \\
1+(\Lambda-1) \exp \left[-\left(\frac{|y|-Y_{0}}{y_{0}}\right)^{2}\right] \\
\quad \times \exp \left[-\left(\frac{z-z_{i}}{z_{0}}\right)^{2}\right], & \text { otherwise }
\end{array}\right.
$$

where $\Lambda$ is the maximum amplitude of the amplification factor, and $y_{0}, z_{0}$ refer to the attenuation scales in the horizontal and vertical directions, respectively, for the variations of $f(y, z)$. The form of attenuation given by $(10)$ indicates that for the region centered at $y=0$ with extension $2 Y_{0}$, the magnitude of the amplification is constant (for a fixed $z=z_{i}$ ) and equal to $\Lambda$; outside this region the magnitude decays exponentially. Note that $f(y \rightarrow x, z \rightarrow x)=1$, which means that far from the center of the irregularity the conductivity of the medium is unaltered. The set of parameters $\left(\Lambda, y_{0}, Y_{(n,}, z_{1}, z_{i}\right)$ defines the influence of the irregularity.

Assuming boundary conditions only related to the fair weather field and the set of parameters for the irregularity $(10,100,0,20,60 \mathrm{~km})$, we get the horizontal electric fields due to the irregularity shown in Fig. 6. The curves of equal value for these fields are plotted automatically. The 'ellipsoidal' dashed curve indicates the irregularity section at a distance of one attenuation scale length from its center. The equipotential curves for the fair weather electric field are also shown. Note that these equipotentials are expulsed from the irregularity.

Figure 7 presents a combined study involving the stratospheric irregularity discussed above and the mapping of ionospheric electric fields, for which a dynamo ionospheric electric field of $-2 \mathrm{mV} / \mathrm{m}$ (westward) and scale length of $2000 \mathrm{~km}$ was assumed. The region of interest is around the origin $y=0$. The ionospheric electric field is mapped downward in about $50 \mathrm{~km}$ with essentially no attenuation (see also Fig. 3). For points near the origin the contour lines of the mapped electric field connect with those of the irregularity. The large scale ionospheric electric ficld originates an asymmetry in the resultant horizontal electric field due to the irregularity in the conductivity 


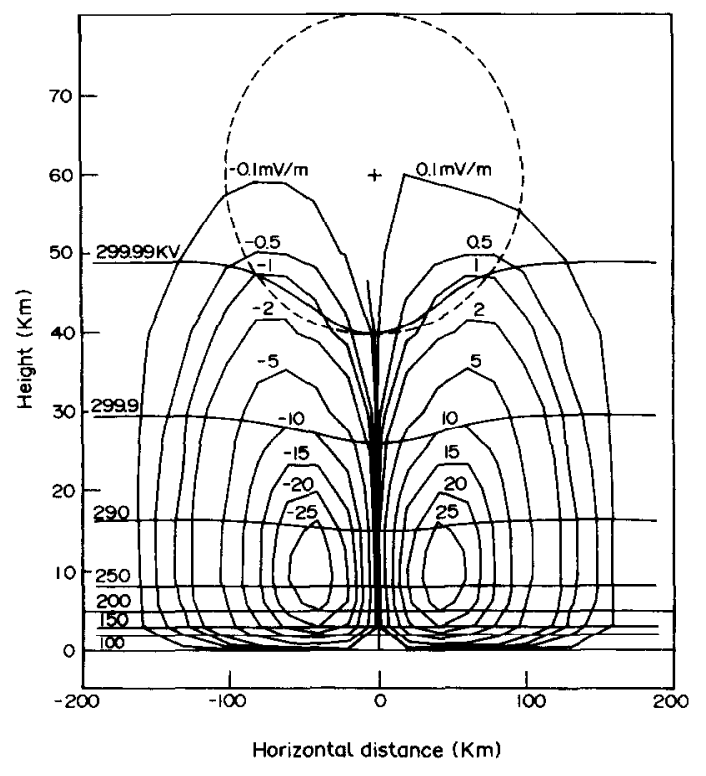

Fig. 6. Curves of equal horizontal electric field values due to a stratospheric irregularity in the electrical conductivity (dashed curve). The equipotential contours are also shown.

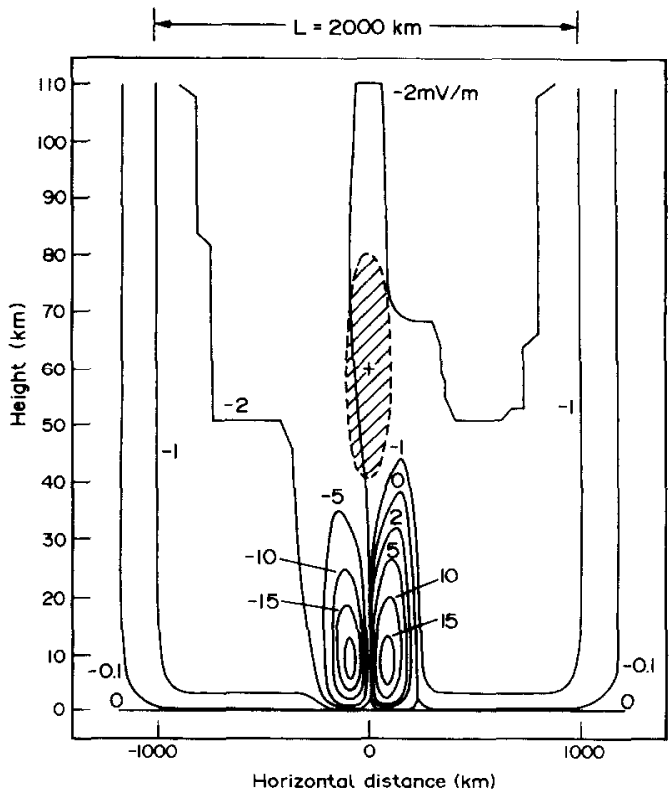

Fig. 7. Curves of equal horizontal clectric field values due to a stratospheric irregularity in the electrical conductivity (dashed curve) and a dynamo ionospheric electric field of $-2 \mathrm{mV} / \mathrm{m}$ and $2000 \mathrm{~km}$ scale length. (essentially svmmetric). At balloon altitudes the magnitude of the mapped horizontal component of the ionospheric electric field is enhanced on the west side $(y<0)$ and attenuated on the opposite east side $(y>0)$ due to the irregularity. Note that the mapped ionospheric field at distances around $y=1000 \mathrm{~km}$ diminishes considerably as discussed before with reference to Fig. 4. For this study the finite difference method was used.

\section{CONCLUSIONS}

From the results presented above we can conclude that ionospheric electric fields at the equator do map down to the lower atmosphere with negligible attenuation when the scale lengths of those fields are of the order of $100 \mathrm{~km}$ or larger. This result is similar to that initially found for high latitudes (MOZER, 1971 ; PARK, 1976). Thus, for these scale lengths, electric field measurements at balloon altitudes of the equatorial atmosphere can be related to the overhead ionospheric electric field (Gonzalez et al., 1982). On the other hand, for scale lengths of the order of tens of kilometers. the ionospheric electric field is strongly attenuated in the equatorial atmosphere, with larger attenuation factors than the corresponding ones for high latitudes. Furthermore, for this range of $L$, it was pointed out by PARK (1979) that a three-dimensional study should be necessary in order to compare measured atmospheric electric ficlds at balloon altitudes with the overhead ionospheric field mapped down to the stratosphere.

It has been also shown that the presence of mountains with a fairly large height as well as a large-scalc conductivity irregularity in the middle atmosphere. such as that expected at the SAMA region during energetic electron precipitation events, can considerably distort the mapped ionospheric electric ficlds at the middle atmosphere.

The present work is a new contribution to the understanding of atmospheric electricity at equatorial latitudes, that can be used as complementary information or even hints in the related experimental research carried out mainly at balloon altitudes.

Acknowledgements - We acknowledge N. B. Trivedi for a critical reading of the manuscript. This work was partially supported by the Fundo Nacional de Desenvolvimento Cientifico e Tecnológico under contract FINEP 537/CT. 


\section{REFERENCES}

Atkinson W., Lunioquist S. and Fahleson U.

Chil Y. T.

COle R. K. and Pierce E. T.

DolezaleK $\mathrm{H}$.

Forbes J. M. and Lindzan R. S.

Gonzales C. A., Kelley M. C., Fejer B. G., ViCKREY J. F. and WOODMAN R. F.

Gonzalez W. D., Dutra S. L. G. and Pinto O. JR

Gonzalez W. D., Pereira A. E. C., Gonzalez A. L. C., Martin I. M., Dutra S. L. G., Pinto O. JR, Wygant J. and Mozer F. S.

Holzworth R. H. and Mozer F. S.

MitChell A. R. and Griffiths D. F.

1980

MOZER F. S.

Ogawa T., Yasuhara M. and Huzita A.

PARK $C$ G.

PAKK C. G.

Park C. G. and Dejnakarintra M.

Richmond A. D. Blanc M., Emery B. A., WAND R. H., Fejer B. G., WoOdman R. F., Ganguly S., Amayenc P., Behnke R. A., Calderon C. and Evans J. V.

SANDSTRÖM A. E.

VOLLAND $H$.

WLos W. L.

WOODMAN R. F.

ZIENKIEWICZ O.C.

Reference is also made to the following unpublished material:

Dejnakarintra M. A.

Dutra S. L. G.

Dutra S. L. G., Pereira A. E. C., Gonzalez A. L. C. and GonZaLES W. D.
1977

1982

1971 The electric field existing at stratospheric elevations as determined by tropospheric and ionospheric boundary conditions. Pure appl. Geophys. 84, 46.

1974 Self-consistent electrostatic field mapping in the high latitude ionosphere. J. geophys. Res. 79, 2790.

1965 Electrification in the Earth's atmosphere for altitudes between 0 and $100 \mathrm{~km}$. J. geophys. Res. 70, 2735

1972 Discussion of the fundamental problem of atmospheric electricity. Pure appl. Geophys. 100, 8.

Atmospheric solar tides and their electrodynamics effects. I. The global $S_{4}$ current system. J. atmos. terr. Phys. 38, 897.

Equatorial electric fields during magnetically disturbed conditions. 2. Implications of simultaneous auroral and equatorial measurements. $J$. geophss. Res. 84, 5803.

Middle atmospheric-electrodynamic modification by solar activity at the South Atlantic Magnetic Anomaly. J. atmos. terr. Phys. 49, 377.

1982 Large horizontal electric fields measured at balloon heights of the Brazilian magnetic anomaly and association to local energetic particle precipitation. Geophvs. Res. Lett. 9, 567.

Direct evidence of solar flare modification of stratospheric electric fields. J. geophys. Res. 84, 363.

1971 Balloon measurements of vertical and horizontal electric fields. Pure appl. Geophys. 84, 32.

1975 Stratospheric horizontal electric fields over mountains. J. atmos. terr. Phys. 37, 841 .

1976 Downward mapping of high latitude ionospheric electric fields to the ground. J. geophys. Res. 81, 168.

1979 Comparison of two-dimensional and three-dimensional mapping of ionospheric electric fields. $J$. geophys. Res. 84, 960 .

1973 Penetration of thunderclond electric fields into the ionosphere and magnetosphere. J. geophys. Res. 78, 6623.

1980 An empirical model of quiet-day ionospheric electric fields at middle and low latitudes. $J$. geophys. Res. $\mathbf{8 5}, 4658$.

1965 Cosmic Ray Physics. North-Holland, Amsterdam.

1972 Mapping of the electric field of the $S_{\varphi}$ current into the lower atmosphere. J. geophys. Res. 77, 1961.

1974 Electric structure of the lower atmosphere. In Structure and Dynamics of the Upper Atmosphere, VERNIANI F. (ed.). Elsevier, Amsterdam.

Vertical drift velocities and east-west electric fields at the magnetic equator. J. geophys. Res. 74, 1205.

The Finite Element Method. McGraw-Hill, London.

A theoretical study of electrical coupling between the troposphere, ionosphere and magnetosphere. Technical Report 3454-3, Radioscience Laboratory. Stanford Electronics Laboratory, Stanford University, Stanford, California.

Campos elétricos em alturas de balão estratosférico. Ph.D. thesis, INPE, São José dos Campos, Brazil.

1985 Atmospheric electric fields: a numerical approach. INPE, São José dos Campos, Brazil. 


\section{APPENDIX}

In problems of atmospheric electricity, equations (1)-(3) can be usually written in the following form for two dimensions:

$$
\mathscr{L} u(\mathbf{x})=0
$$

with

$$
\mathscr{L}=A \frac{\partial^{2}}{\partial x^{2}}+C \frac{\partial^{2}}{\partial y^{2}}+D \frac{\partial}{\partial x}+E \frac{\partial}{\partial y}+F
$$

where $A, C, D, E$ and $F$ can be functions of the independent variables $x$ and $y$ within the region of interest. This appendix describes briefly the numerical approach to solve equation (1)-or (Al) - with boundary condition of the Dirichlet type.

\section{Method of finite differences}

The equation (7) can be written as

$$
A \frac{\partial^{2} u}{\partial p^{2}}+C \frac{\partial^{2} u}{\partial z^{2}}+D \frac{\partial u}{\partial p}+E \frac{\partial u}{\partial z}=0
$$

where $p=\arctan (\alpha y)$ so that we have $y \rightarrow \pm \infty$ when $p \rightarrow$ $\pm \pi / 2$, and $u(p, z)$ is the electric potential. The bi-dimensional region of interest $\left(-\pi / 2 \leqslant p \leqslant \pi / 2,0 \leqslant z \leqslant z_{m}\right)$ is substituted by a finite set of points, discretized with $M+2$ and $N+2$ points in the $p$ and $\angle$ directions, respectively.

Then at a grid point $P$ we find a linear relationship between the value of $u$ there and at the neighboring points, repeating this procedure for the other points of the grid (e.g. MiTCHELL and GRIFFITHS, 1980). We have an approximate solution $u_{i j}$ to the solution $u(p, z)$ at the grid points $P\left(p_{j}, z_{j}\right)$, where $p_{i}=(-\pi / 2)+i \pi /(M+1)$, for $i=0,1,2, \ldots, M+1$ and $z_{j}=j z_{m} /(N+1)$, for $j=0,1,2, \ldots, N+1$. This procedure produces the following finite difference approximation to (A2) :

$$
P_{i} \mathbf{u}_{i-1}+Q_{i} \mathbf{u}_{i}+T_{i} \mathbf{u}_{i+1}=\mathbf{r}_{i}
$$

for $i=1,2,3, \ldots, M$, where $P_{i}$ and $T_{i}$ are diagonal matrices of order $N, Q_{i}$ is a tridiagonal matrix of the same order, and the vectors $\mathbf{u}_{i}=\left[u_{i 1}, u_{i 2}, \ldots, u_{i N}\right]^{T}$ (the symbol ${ }^{T}$ stands for transpose) and $\mathbf{r}_{i}$ have $N$ elements each.

In a compact notation, (A3) can be expressed by $Z \mathbf{U}-\mathbf{R}$, where $Z$ is a block-tridiagonal matrix (BTM) of order $M$, and $\mathbf{U}=\left[\mathbf{u}_{1}, \mathbf{u}_{2}, \ldots, \mathbf{u}_{M}\right]^{T}$ and $\mathbf{R}$ are block-vectors with $M$ elements each. The solution $\mathbf{U}=Z^{-1} \mathbf{R}$ can be obtained by the classical algorithm of Gauss elimination applied to BTM systems. Note that $\mathbf{u}_{0}$ and $\mathbf{u}_{M+1}$, together with first and last elements of $\mathbf{r}_{i}$, contain the boundary conditions.

\section{Method of finite elements}

This method (e.g. ZIENKIEwICZ, 1977) uses a subdivision of the region of interest in sub-regions e called finite elements. The vertex of each element (grid points) are called nodes which are globally numbered over all the region of interest They are also numbered locally (at each element).

A solution for equation (A1) can be obtained considering, initially, that within each element $e$ an approximate solution $u(\mathbf{x})$ varies as:

$$
u(\mathbf{x})=\sum_{j=1} u_{k(j)}^{\iota} N_{j}^{u}(\mathbf{x})
$$

where $f$ is the number of nodes of the element $e^{2}, u_{k(j)}^{c}$ is the approximate value $u$ at the node $j$ of element $e$ (corresponding to the global numbering $k$ ), and $N_{i}(\mathbf{x})$ is an interpolation function of node $j$ (that has value 1 at this node and value 0 at the other nodes of the element or outside it). In general, equation (A4) does not exactly satisfy equation (A1), giving rise to a local residue $r(\mathbf{x}) \equiv \mathscr{L} u(\mathbf{x})$, where $\mathscr{L} \equiv \nabla \cdot(\tilde{\sigma} \cdot \nabla)$ and $u$ is the electric potential.

The problem reduces to finding the values $u_{k}$ that cancel the total residue. Namely, the sum of the local residues within the whole region of interest, weighted by appropriate functions $\omega_{i}(\mathbf{x})$, has to be zero:

$$
\sum_{\varepsilon} \int_{i} N_{i}^{e} \nabla \cdot(\tilde{\sigma} \cdot \nabla u) \mathrm{d} \boldsymbol{V}=0 .
$$

In this expression $\omega_{i}(\mathbf{x})=N_{i}^{e}(\mathbf{x})$; this choice is known as the Galerkin choice. We can also show that:

$$
\sum_{i} \sum_{j=1}^{\prime} a_{i j} u_{(j)}^{c}=0
$$

where

$$
a_{i j}=\int_{i} \nabla N_{i}^{e} \cdot\left(\tilde{\sigma} \cdot \nabla N_{i}^{\prime}\right) \mathrm{d} V
$$

can be calculated using the theory of isoparametric elements (ZIENKIEWICZ, 1977).

Combining the double sum of (A5) with the boundary conditions we finally get :

$$
\sum_{k} A_{i k} u_{k}=B_{i}
$$

where $A$ is a band-type matrix with a width that depends on the numbering of the nodes and with an order given by the total number of nodes. A description of the vectors, matrix and their elements that show up in this appendix is given by Dutra (1982). 